\title{
COMPORTAMIENTO FORRAJERO DE BRACHIARIA DECUMBENS PASTOREADA CON OVINOS PELIBUEY A DIFERENTES NIVELES DE ASIGNACIÓN EN EL TRÓPICO HÚMEDO DEL ECUADOR
}

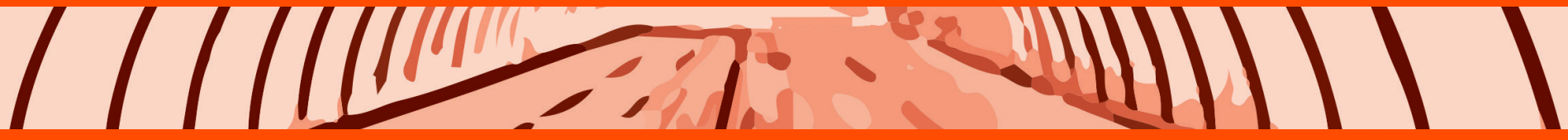

Autores:

Mildred Rivera-Acurio ${ }^{1}$

Eugenia Cienfuegos-Rivas ${ }^{1,2^{*}}$

José Espinosa Marroquín ${ }^{1}$

Pedro Zárate ${ }^{2}$

Martín Ibarra²

Jonathan Garay Martínez 


\section{Resumen}

El presente estudio se realizó con el fin de determinar el efecto de la asignación de forraje sobre la producción de materia seca (MS) en praderas de Brachiaria decumbens bajo el pastoreo rotacional con ovejas Pelibuey en la época seca en el trópico húmedo del Ecuador. Se evaluaron las asignaciones forrajeras de 5, 7 y 9\% del peso vivo de los animales en dos periodos de rebrote de 24 días cada uno. Se utilizaron nueve parcelas de 345,15 m2 pastoreadas por tres días. Para cada asignación se utilizaron ocho ovejas de la raza Pelibuey con un peso promedio individual de $35 \mathrm{~kg}$ y una edad promedio de 18 meses. Para determinar la producción de forraje se tomaron muestras del pasto al inicio y final del pastoreo. Los datos obtenidos fueron evaluados con un diseño experimental de parcelas divididas, con las asignaciones en la parcela grande y el periodo de rebrote (PR) en la parcela pequeña. La producción de MS de la parte aérea, tallos, láminas foliares y vainas con el 9\% de asignación forrajera fue mayor en el primer PR; la proporción de vainas con el 9\% de asignación fue mayor en el segundo PR; y la producción acumulada de la parte aérea de la planta y sus órganos en los dos periodos de rebrote fue igual $(P>0,05)$ entre las tres asignaciones. Se concluye que, las asignaciones forrajeras con 24 días de recuperación de la pradera modificaron la producción de MS de forraje durante los dos periodos de rebrote en la época seca.

Palabras clave: Asignación forrajera, Brachiaria decumbens, Ecuador, ovinos Pelibuey

\section{Abstract}

The present study was conducted to determine the effect of herbage allowance on production of dry matter (MS) in Brachiaria decumbens pastures under rotational grazing with Pelibuey ewes in the dry season in the humid tropics of Ecuador. The forage allowance of 5,7 and $9 \%$ of the live weight of the animals was evaluated in two periods of regrowth of 24 days each. There were nine experimental plots of $345.15 \mathrm{~m} 2$ grazed for three days. For each allowance eight Pelibuey ewes were used with an average individual weight of $35 \mathrm{~kg}$, and an average age of 18 months. Grass samples were taken to determine the production of forage at the beginning and end of the grazing. The data were evaluated with a split plot design, with the herbage allowance in the main plot and the period of regrowth (PR) in the small plot and a complete block design to evaluate the effect random allocations in cumulative production forage in both periods. The dry matter production of the aerial part, stems, leaf blades and sheaths with $9 \%$ forage allocation was higher in the first period of regrowth, the proportion of pods with $9 \%$ allocation was higher in the second period of regrowth, and the combined production of the aerial part of the plant and its organs in the PR was similar with all three assignments. It was concluded that, forage allocations with 24 days of recovery modified the prairie dry matter production of forage during the two PR during the dry season.

Keywords: Allocation forage, Brachiaria decumbens, Ecuador, Pelibuey 
Comportamiento Forrajero de Brachiaria decumbens pastoreada con ovinos Pelibuey a diferentes niveles de asignación en el trópico húmedo del Ecuador

\section{Introducción}

En Ecuador hay aproximadamente 11.903 .873 ha clasificadas para uso agropecuario. En la región Costa del total nacional, el 70 \% corresponde a cultivos permanentes, el 45,3\% a pastos cultivados, el 17,14 a pastos naturales, el 1,07\% a páramos y el 27,66 \% a montes; mientras que, en la provincia de Santo Domingo de los Tsáchilas del total de la Costa el 9,06 y 4,35\% son superficies con pastos cultivados y pastos naturales. De igual forma en el país se tiene un censo bovino nacional de 300.545 toros y 1.995.694 vacas en la categoría de dos o más años de edad, de los cuales, en la región Costa se encuentran el 24,6 y $34,9 \%$ respectivamente y en la provincia de Santo Domingo de los Tsáchilas se tienen registrados el 10, $39 \%$ de toros y el 8,08\% de vacas. En cuanto a los ovinos se refiere, en el Ecuador se tiene un censo nacional de 126.655 ovinos menores de seis meses y 585.041 mayores de seis meses de los cuales cerca del $96 \%$ se encuentran en la Sierra y el resto en la Costa (INEC, 2012). Lo anterior es un indicador del potencial pecuario del país, debido a que cerca del 50\% de las tierras cultivadas se utilizan para producir forraje, el principal alimento de rumiantes.

La eficiencia biológica y de producción de las plantas forrajeras está determinada por su potencial genético y por el ambiente. Para alcanzar dicho potencial, el manejo de la pradera es fundamental, porque la eficiencia de la producción animal depende de la cantidad y la calidad del forraje producido para su alimentación (Rojas-Hernández et al., 2011; Jailson et al., 2005). La interacción entre el animal y la producción de forraje de una pradera se debe de considerar al determinar la carga animal, porque el animal afecta la disponibilidad, estructura y valor nutritivo del forraje durante el pastoreo, y porque la producción y calidad del forraje determina la productividad de los animales (Del Pozo, 2002).

Una forma de optimizar el pastoreo es lograr el equilibrio entre la producción de materia seca de la pradera y el requerimiento nutricional del animal (Faría, 2006), manipulando la asignación de forraje a ser consumido por los animales en la superficie disponible para el pastoreo (Faría, 2006; Hernández-Mendo et al., 2000; Murillo, 1999) y considerando el periodo de reposo entre dos pastoreos sucesivos para que la planta forrajera acumule reservas de carbohidratos en sus raíces y forme una gran cantidad de masa verde (Berton y Richter, 2011; Faría, 2006).

El potencial genético de los pastos del género Brachiaria ha sido estudiado en ambientes con clima tropical donde el forraje es la principal fuente de alimentación de los animales (Mena-Urbina et al., 2007), particularmente el cultivar Brachiaria decumbens Stapf que se ha adaptado a diversidad de suelos (ácidos, calcáreos y pedregosos) y es resistente al pastoreo (Olivera et al., 2006) y es utilizado en la alimentación del ganado por su buen rendimiento de biomasa en la época seca y por su moderado valor nutritivo (Vázquez et al., 2010; Olivera y Machado, 2004; Passoni et al., 1992).

Por otro lado, se considera que las ovejas de pelo de la raza Pelibuey son una alternativa viable para los ganaderos en los trópicos de América; ya que estos animales se adaptan a condiciones de alta temperatura y humedad, y son resistentes a enfermedades. Esta raza de ovinos pueden consumir en promedio un $4,2 \%$ de su peso vivo en MS de forraje por lo que no se requieren de grandes extensiones de tierra para satisfacer sus requerimientos nutricionales (Gutiérrez y Díaz, 2008). Por lo anterior el objetivo de la presente investigación fue determinar el efecto de la asignación de forraje a ovinos Pelibuey sobre el comportamiento forrajero de B. decumbens en un sistema de pastoreo rotacional.

\section{Materiales y Métodos}

Esta investigación se condujo durante la época seca del año 2012 en la granja Experimental El Oasis, propiedad de la Universidad Tecnológica Equinoccial, Extensión Santo Domingo. La granja está ubicada en el recinto Chila, provincia de Santo Domingo de los Tsáchilas, a $0^{\circ} 13,29^{\prime}$ de latitud sur, $79^{\circ} 15,83^{\prime}$ de longitud oeste y a 416 msnm, con un clima tropical húmedo, caracterizado por una 
temperatura media anual de $24,3{ }^{\circ} \mathrm{C}$, precipitación anual de 2600 $2800 \mathrm{~mm}$, distribuidos unos $2200 \mathrm{~mm}$ en la época lluviosa (enero a junio) y unos $430 \mathrm{~mm}$ en la época seca (julio a diciembre).

Parcelas Experimentales. Cada parcela experimental se ubicó en una pradera establecida de $B$. decumbens de seis años de edad, con dimensiones de 29,5 m de largo y 11,7 m de ancho (345,15 m2). El área total del experimento, incluyendo pasillos de manejo, fue de 10.000 m2. Las parcelas se delimitaron con malla campera y se agruparon en 3 bloques ( $A, B$ y $C$ ), cada uno de ellos conteniendo nueve divisiones. Los bloques se dividieron en cuadrantes I, II y III, que contuvieron tres parcelas experimentales donde se dispuso al azar las asignaciones ( 5 , 7 y $9 \%$ del peso vivo del total de kilos totales de animal en pastoreo). Los tres bloques sostuvieron una ronda de pastoreo empezando con el cuadrante I del bloque A, el I del B y así sucesivamente hasta terminar con el III del C (Figura 1).

Sistema de rotación. El sistema de rotación consistió en el pastoreo de 24 ovejas Pelibuey divididas en tres grupos de ocho, por tres días consecutivos en cada parcela de los bloques A, B y C con periodos de rebrote de 24 días. El área de cada parcela se calculó tomando en cuenta el peso total de las ovejas en pastoreo y la producción de forraje al inicio de cada pastoreo. Los tratamientos (asignación de forraje) se distribuyeron aleatoriamente en las parcelas, al inicio de cada periodo de ocupación (Gutiérrez y Díaz, 2008; Hernández et al., 2002; Hernández-Mendo et al., 2000).

Producción de forraje. El forraje de cada parcela se muestreó por las tardes cortando con una hoz la parte aérea de la pastura a $2 \mathrm{~cm}$ de altura del suelo, a la entrada y salida de cada pastoreo (Pueyo et al., 2008). Para estimar la producción de forraje se tomaron dos muestras aleatorias de 0,25 m2 cada una en cada rotación de pastoreo. De cada muestra se tomaron seis plantas al azar, separándose tallo, láminas y vainas (Rincón-Carruyo et al., 1997) para determinar la materia seca de cada órgano y la materia seca total (MST). Se calculó la producción de forraje de la parte aérea, tallos, láminas foliares y vainas en los periodos de rebrote (Paladines, 1992) en t ha-1 (ecuación 1) y la producción de la
MST para cada periodo de rebrote o producción de forraje disponible para pastoreo (ecuación 2) después de 24 d de descanso.

$$
\mathrm{P}=\left(\mathrm{Pm}+\mathrm{Pm}_{2}\right) 20
$$

Donde, $\mathrm{P}=$ Producción de materia seca total, $\mathrm{t}$ ha-1; $\mathrm{Pm}=$ Peso seco de las sub-muestras uno y dos, $\mathrm{kg}(0,25 \mathrm{m2})-1$.

$$
P_{i}=P E_{i+1}-P S_{i}
$$

Donde, $\mathrm{P}_{\mathrm{i}}=$ Producción de materia seca total para cada periodo de rebrote, $t$ ha-1 $(i=1,2) ; P E$ = Producción a la entrada del pastoreo, $\mathrm{t} \mathrm{ha}^{-1}$; PS = Producción a la salida del pastoreo, $\mathrm{t} \mathrm{ha}{ }^{-1}$.

Producción acumulada de forraje. Se calculó la producción acumulada de la parte aérea, tallos, láminas foliares y vainas (ecuación 3).

$$
\mathrm{PA}_{\mathrm{i}}=\sum \mathrm{P}_{\mathrm{i}}
$$

Donde, PA = Producción acumulada de forraje de la parte aérea, tallos, láminas foliares y vainas, $\mathrm{t} \mathrm{ha}^{-1}(\mathrm{i}$ = periodos de rebrote 1 y 2) y $\mathrm{P}_{\mathrm{i}}$ de la ecuación 2.

Proporción de órganos. Se calculó (ecuación 4) la relación entre tallos, láminas foliares y vainas con el total de producción de forraje de la parte aérea (Carrilho et al., 2012).

$$
\mathrm{PO}_{\mathrm{i}}=\mathrm{Om}_{\mathrm{i}} / \mathrm{T} 100
$$

Donde, $\mathrm{POi}=$ Proporción de materia seca de los órganos, \%; Omi = Peso promedio de cada órgano ( $\mathrm{i}=$ tallos, hojas, vainas), $\mathrm{g}$ muestra ${ }^{-1} ; \mathrm{T}=$ Peso total promedio de los órganos, g muestra ${ }^{-1}$.

Requerimiento de forraje. Se calculó el forraje necesario para el total de kilogramos animal pastoreado por parcela según las asignaciones teniendo en cuenta las ecuaciones sugeridas por Lemus (2012).

$$
R T=X P A * A s g * 0,01
$$


Comportamiento Forrajero de Brachiaria decumbens pastoreada con ovinos Pelibuey a diferentes niveles de asignación en el trópico húmedo del Ecuador

Donde, RT = Requerimiento total de los ovinos de materia seca, $\mathrm{kg}$; PA = Peso promedio vivo de los ovinos, kg; Asg = Asignaciones de forraje, 5,7 y $9 \%$.

$$
\mathrm{PMS}=\mathrm{PMV}^{*} \mathrm{MS}^{*} 0,01
$$

Donde, PMS = Producción de materia seca del pasto, $\mathrm{kg}(\mathrm{m} 2)$ 1; PMV = Producción de materia verde del pasto, $\mathrm{kg}(\mathrm{m} 2)-1 ; \mathrm{MS}=$ Materia seca, \%.

$$
\mathrm{AP}=\mathrm{RT} / \mathrm{PMS}
$$

Donde, AP = Área de pastoreo, $\mathrm{m}^{2}$.

Análisis Estadístico. Se utilizó un diseño de parcelas divididas, con las asignaciones como la parcela grande y los periodos de rebrote como la parcela pequeña y la prueba de comparación de medias de Tukey con $\mathrm{a}=0,05$.

$$
Y_{i j k}=\mu+B_{i}+A_{i}+E_{(i i)}+P_{k}+(P A)_{j k}+E_{i k k 1}
$$

Donde, $\mathrm{Y}_{\mathrm{ij} / \mathrm{kl}}$ = l-ésima observación de la k-ésima asignación en el j-ésimo periodo del i-ésimo bloque; $u_{i}=$ media general; $B_{i}=$ efecto del i-ésimo bloque; $A_{j}=$ efecto del j-ésimo porcentaje de asignación; $E_{(i j)}=$ Error $A ; P_{k}=$ Efecto del k-ésima periodo de rebrote; $(P A)_{j k}=$ Efecto de la interacción entre porcentaje de asignación y periodo de rebrote; $\mathrm{E}_{\mathrm{ijkl}}=$ Error aleatorio.

\section{Resultados y Discusión}

\section{Efecto de las asignaciones en la producción de la pradera durante los periodos de rebrote}

En el primer periodo de pastoreo, la cantidad de forraje presente a la entrada de los animales varió de 0,71 a 5,65 t ha-1, pero no se analizó el efecto de las asignaciones porque las parcelas aun no fueron pastoreadas. Para el segundo periodo de rebrote (PR), se observó que con la asignación forrajera de 9\% la producción de materia seca de la parte aérea, láminas foliares y vainas fue menor $(P \leq 0,05)$, observándose una disminución entre $60,4 \%$ para la parte aérea y $64,6 \%$ para las vainas (Cuadro 1). Cabe señalar también que la producción de la parte aérea, tallos, láminas foliares y vainas disminuyó, sin considerar una asignación en particular, un 63,1\% para las láminas foliares y $67,3 \%$ para los tallos. Contrario a lo observado para la asignación de 9\%, no hubo diferencias significativas ( $P>0,05)$ en la producción de la parte aérea y de sus órganos en el primer y segundo PR por efecto del 5 y 7\% de asignación forrajera; sin embargo, fue menor la producción de la parte aérea y vainas (66,8\%), tallos $(73,6 \%)$ y láminas foliares (63,5\%) en el segundo PR (Cuadro 1).

Las asignaciones forrajeras, sin considerar un PR en particular, tuvieron efectos iguales $(P>0,05)$ en la producción de forraje; no obstante, con el 9 \% de asignación la producción de la parte aérea $\left(0,67 \mathrm{t} \mathrm{ha}^{-1}\right)$, tallos $\left(0,13 \mathrm{tha}^{-1}\right)$, hojas $\left(0,36 \mathrm{t} \mathrm{ha}^{-1}\right)$ y vainas $\left(0,18 \mathrm{t} \mathrm{ha}^{-1}\right)$ fue mayor (25,4, 15,4 y $27,8 \%$ hojas y vainas) que con el 5 y $7 \%$ de asignación (Figura 2).

Con el 9\% de asignación la mayor cantidad de MS de la parte aérea, hojas y vainas producidas en el primer PR indican que la pradera tuvo mayor resistencia a la presión de pastoreo, lo que permitió a las plantas de $B$. decumbens tener más reservas de carbohidratos, más área foliar remanente que les permitió realizar mayor cantidad de fotosíntesis, y por tanto mayor rebrote (Cruz, 2010; del Pozo, 2002; Marchegiani, 1985). Con el 5 y 7\% de asignación la producción igual en el primer y segundo PR de la parte aérea, láminas foliares y vainas indica que la pradera resistió de igual forma en ambos PR. El anterior comportamiento fue observado por Ramírez et al. (2003) al evaluar asignaciones forrajeras desde 3,0 hasta 7,5\% en praderas de estrella (Cynodon plectostachius) asociada con clitoria (Clitoria ternatea) en seis pastoreos en la época lluviosa con bovinos, donde se reporta que con asignaciones de 3,0 y 4,5\% la producción de forraje presente fue menor al aumentar los pastoreos, mientras que con las asignaciones de 6,0 y $7,5 \%$ se mantuvo la producción original de forraje disponible al inicio del pastoreo.

La cantidad de hojas (láminas foliares más vainas) y parte aérea en el primer PR fue de 27,1 a 34,2 kg ha ${ }^{-1}$ día $^{-1}$, mientras que en el 
segundo PR fue solamente de 10,1 y 12,1 kg ha-1 día ${ }^{-1}$, que son similares a los reportados por Fisher y Kerridge (1998) para la parte aérea del pasto B. decumbens $\left(24,8 \mathrm{~kg} \mathrm{ha}^{-1} \mathrm{día}^{-1}\right)$ en la época seca, lo que indica que las asignaciones con un PR de 24 días en la época seca fue muy corto para la producción de hojas y la parte aérea; como lo reportan Fernández et al. (2012) al evaluar la producción de forraje presente en praderas de B. humidicola en la época seca con PR de 7 a 49 días (intervalos de 7 días), quienes concluyen que las cantidades de forraje presente a partir de 21 hasta 42 días de rebrote fue de 4,8 hasta 28,6 kg ha ${ }^{-1}$ día $^{-1}$ y para las hojas de 4,8 hasta $14,3 \mathrm{~kg} \mathrm{ha}^{-1}$ día $^{-1}$.

\section{Producción acumulada de forraje producido}

Para la producción acumulada del forraje producido en las praderas no hubo diferencias significativas $(P>0,05)$ en la producción acumulada de forraje total por efecto de las asignaciones; sin embargo, con el 9\% de asignación la producción acumulada de la parte

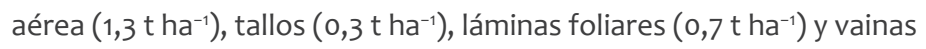
$\left(0,4\right.$ t ha $\left.^{-1}\right)$ fue mayor en un 24,$6 ; 15,4 ; 26,4$ y $27,8 \%$ respectivamente que con el 5 y 7 \% de asignación (Figura 2).

La disminución en la producción acumulada de forraje por efecto de las menores asignaciones ( 5 y $7 \%$ ) pudo haber sido por una defoliación por efecto de la época seca que disminuyó la capacidad de los tallos para sostener nuevos rebrotes, el área foliar remanente y las reservas de carbohidratos como lo reportan Martínez et al. (2008) quienes al evaluar durante la época seca (4 meses) en la B. humidicola el efecto de alturas de corte concluyen que al inicio de la época seca la producción por 48 días (igual a los dos PR de esta investigación) con las alturas de 9, 12 y $15 \mathrm{~cm}$ fue de 0,48, 0,43 y 0,67 tha $\mathrm{t}^{-1}$, respectivamente mientras que al final de la época seca fue de 0,19, 0,19 y 0,29 t ha-1.

\section{Efecto de las asignaciones en el porcentaje de los órganos de la parte aérea de la planta}

Con el 9\% de asignación forrajera el porcentaje de vainas en el forraje de la parte aérea aumentó $(P \leq 0,05) 3,4 \%$ en el segundo PR (Cuadro 2).
El porcentaje de vainas fue igual $(\mathrm{P}>0,05)$ para todas las asignaciones en el primer y segundo PR; sin embargo, se observó que a partir del $5 \%$ de asignación el porcentaje de vainas fue 0,8 y 1,9\% mayor en el primer y segundo PR, respectivamente (Cuadro 2).

El efecto de las asignaciones en el porcentaje de tallos y láminas foliares en el primer y segundo PR fue igual $(P>0,05)$. No obstante, el porcentaje de tallos en el segundo PR (19,5\%) con el 7\% de asignación fue 1,7\% menor, y con el 9\% de asignación fue 3,1 y 1,6\% menor en el primer y segundo PR, respectivamente. El porcentaje de láminas foliares fue 2,1, 1,2 y $3,4 \%$ menor en el segundo PR, respectivamente, con el 5 (54,6\%), 7 $(52,4 \%)$ y $9 \%(51,9 \%)$ de asignación forrajera (Figura 3$)$.

En ambos PR, sin una asignación en particular, el porcentaje de tallos, láminas foliares y vainas fue igual $(P>0,05)$. Sin embargo, en el segundo PR el porcentaje de tallos (18,9\%) y láminas foliares (53,0\%) fue 0,3 y 2,2\% menor, respectivamente (Figura 4); y el de vainas $(28,2)$, 2,5\% mayor (Cuadro 2) con respecto al primer PR.

Con las asignaciones forrajeras, sin un PR en particular, el porcentaje de tallos, láminas foliares y vainas fue igual $(P>0,05)$. No obstante, el porcentaje de tallos fue 2,0 y $2,5 \%$ mayor con el $7 \%$ (20,3\%) que con 5 y $9 \%$ de asignación, respectivamente; el de láminas foliares, $2,4 \%$ mayor con el $5 \%$; y el de vainas fue $1,4 \%$ mayor a partir del $5 \%$ de asignación (25,7\%) (Figura 3).

La mayor proporción de vainas en el segundo PR con el 9\% de asignación indica que las ovejas seleccionaron las láminas foliares a las vainas por su mayor digestibilidad, contenido de proteína y menor fibra que las vainas; por tanto, las vainas se acumularon en mayor proporción en el segundo PR a pesar de que disminuyeron en cantidad (Rodrigues, 2012; González et al., 2003). Además, de que el factor de la época seca pudo influir, ya que al restringirse el crecimiento de tallos por la deficiencia de agua, hubo mayores cantidades de vainas y láminas foliares, como lo indican Fernández et al. (2012), quienes concluyeron que la proporción de hojas de B. humidicola aumentó en la época seca de 51,7 a 68,0\% al cambiar el PR de 42 a 21 días (7 días de intervalo), respectivamente. 
Comportamiento Forrajero de Brachiaria decumbens pastoreada con ovinos Pelibuey a diferentes niveles de asignación en el trópico húmedo del Ecuador

De haber existido diferencias en la proporción de vainas por efecto de las asignaciones, la menor proporción observada de vainas a medida que la asignación forrajera fuera menor, indicaría que con menores asignaciones la pradera fue consumida en exceso (hubo consumo de vainas) y el área foliar remanente disminuiría al igual que sus reservas de carbohidratos, provocando de esta forma una menor proporción de rebrotes de las vainas (González et al., 2003; Beguet y Bavera, 2001).

Si la proporción de las láminas foliares con el 5\% de asignación hubiera sido mayor que con el 7 y 9\%, esto indicaría que los meristemos apicales fueron consumidos en exceso reduciéndose posiblemente la altura de rebrote y la pradera para recuperarse tendría que producir más láminas foliares porque es en este órgano donde mayoritariamente podían distribuirse los carbohidratos desde las raíces (Bernal y Espinosa, 2003; Beguet y Bavera, 2001).

\section{Conclusiones}

La asignación de forraje entre 5 y 9\% no modificó la producción de la parte aérea, láminas foliares, vainas y la proporción de vainas en praderas de Brachiaria decumbens en la época seca.

Con cualquier asignación forrajera la producción de forraje fue modificada en ambos periodos de rebrote.

La producción acumulada de forraje de la parte aérea, tallos, láminas foliares y vainas en los periodos de rebrote no fue modificada por las asignaciones forrajeras.

\section{Agradecimientos}

Se agradece a SENESCYT-Ecuador por el apoyo brindado a la UTE a través del Proyecto PROMETEO VIEJOS SABIOS. Al soporte técnico y económico prestado por la Universidad Tecnológica
Equinoccial, Campus Santo Domingo y a la Facultad de Ingeniería y Ciencias de la Universidad Autónoma de Tamaulipas, México por las facilidades otorgadas para hacer posible esta investigación.

\section{Referencias Bibliográficas}

Beguet, H.A., y Bavera, G.A.. 2001. Fisiología de la planta pastoreada. Curso de Producción Bovina de Carne, Facultad de Agronomía y Veterinaria, Universidad Nacional de Rio Cuarto. Rio Cuarto provincia de Córdova, República de Argentina. (FAV UNRC). Consultado el 14 de agosto del 2012. http://www.produccionbovina.com/produccion_y_manejo_ pasturas/pastoreo\%20sistemas/04-fisiologia_de_la_planta_ pastoreada.pdf.

Bernal, J., y Espinosa, J. 2003. Manual de nutrición y fertilización de pastos. International Plan Nutrition Institute (IPNI). p 1-94.

Berton, C.T., y Richter, E.M. 2011. Referências Agroecológicas Pastoreio Racional Voisin (PRV). Nucleo de Pastoreio Racional Voisini UFSC. Governo do Estado Do Paraná. CEPRA, Curitiba, Brasil. consultado el 20 de julio del 2013. http://www.cpra.pr.gov.br/ arquivos/File/CartilhaPRV.pdf

Carrilho, P.H.M, Alonso, J., Santos, L.D.T., y Sampaio, R.A. 2012. Comportamiento vegetativo y reproductivo de Brachiaria decumbens vc. Basilisk bajo diferentes niveles de sombra. Revista Cubana de Ciencia Agrícola 46(1):85-90. Consultado el 20 de julio del 2013. http://www.redalyc.org/articulo. oa?id=193024313014

Cruz, H.A. 2010. Dinámica de crecimiento y valor nutritivo del pasto mulato, a diferente manejo de pastoreo. Colegio de Posgraduados. Tesis de Doctorado, Montecillo, México.

Del Pozo, R.P.P. 2002. Bases ecofisiológicas para el manejo de los pastos tropicales. PASTOS, XXXII (2), 109 - 137

Faría, M.J.(2006). Manejo de pastos y forrajes en la ganadería de doble propósito. X Congreso Venezolano de Zootecnia, X Seminario Manejo y Utilización de Pastos y Forrajes en Sistemas de Producción Animal. LUZ-FCV, Maracaibo del 20 al 22 de abril 
del 2006. X Seminario de Pastos y Forrajes. 1-16 http://www. avpa.ula.ve/congresos/seminario_pasto_X/Conferencias.html

Fernández, J.L, Gómez, I., y Cordoví, E. 2012. Efecto de la edad de rebrote en el rendimiento y contenido proteico del pasto Brachiaria humidicola cv CIAT-609 en un suelo vertisol. Rev. Prod. Anim. 24(1):1-7.

Fisher, M.J., y Kerridge P.C.1998. Agronomía y fisiología de las especies de Brachiaria. En Miles, J.W., Maass, B.L., y do Valle, C.B. (ed.). 1998. Brachiaria: Biología, agronomía y mantenimiento. No. 295. CIAT, Colombia.p.46-57.

González, M.S.J.C., Améndola, M.R.D., Martínez, H.P.A., y Burgueño F.J.A. 2003. Pastoreo mixto de borregas y vaquillas. En Asoc. Mexicana de Técnicos Especialistas en Ovinicultura (ed.), Curso de alimentación en bovinos, Pachuca, México. 24 y 25 de abril de 2003. p. 1-27.

Gutiérrez, O.E., y Díaz, S.H.. 2008. Estime cuántas unidades animal mes (UAM) tiene en su rancho para determinar la adecuada capacidad de carga. Consultado el 18 de septiembre del 2012. Ebookbrowse http://ebookbrowse.com/031-estime-cuantasunidades-animal-mes-uam-tiene-su-rancho-para-determinarla-adecuada-capacida-pdf-d115677037

Hernández, G.A., Martínez, H.P.A., Mena M.U., Pérez J.P, y Enríquez Q.J.F.2002. Dinámica del rebrote en pasto insurgente(Brachiaria brizantha Hochst. Stapf.) pastoreado a diferente asignación en la estacón de lluvias. Téc. Pecu. Méx. 40(2):193-205.

Hernández-Mendo, O., Pérez-Pérez, J., Martínez-Hernández, P.A., Herrera-Haro, J.G., Mendoza-Martínez, G.D., y HernándezGaray, A. 2000. Pastoreo de kikuyo (Pennisetum clandestinum Hochts.) por borregos en crecimiento a diferentes asignaciones de forraje. Agrociencia 34(2):127-134.

INEC (Instituto Nacional de Estadísticas y Censos). 2012. Visualizador de estadísticas Agropecuarias del Ecuador ESPAC para el año 2010. El documento fue consultado el 20 de julio del 2013. http://www.inec.gov.ec/estadisticas/?option=com_content\& view $=$ article $\&$ id $=103 \&$ Itemid $=75$

Jailson L.F., Miranda da Fonseca, D., Gomide, J.A., do Nascimento
Junior, D., Teixeira V.C.M., Vieira de Morais, R., Mistura, C., da Cunha Reis, R., y Azevedo Martuscello, J. 2005. Acúmulo de forragenem pastos de Brachiaria decumbens adubados com nitrôgenio. Pesq. Agropec. Bras. 40(4):397-403.

Lemus, G.V. 2012. Manual de prácticas producción de leche en pastoreo de praderas de clima templado. Universidad Nacional Autónoma de México consultado el 7 de noviembre del 2012. http://www.fmvz.unam.mx/fmvz/licenciatura/coepa/ archivos/Manuales/9_PRODUCleche_CLIMAtemplado.doc.

Marchegiani, G. 1985. Morfofisiología de plantas forrajeras. Cuaderno de Actualización Técnica 36:6-16. Facultad de Agronomía y Veterinaria, Universidad Nacional de Rio Cuarto. Rio Cuarto provincia de Córdova, República de Argentina. consultado el 20 de julio del 2013. http://www.produccion-animal.com. ar/produccion_y_manejo_pasturas/pastoreo\%20sistemas/31morfofisiologia_plantas_forrajeras.pdf

Martínez, M.D., Hernández Garay, A., Enríquez Quiroz, J.F., Pérez Pérez, J., González Muñoz, S.S:, Herrera Haro, J.G. 2008. Producción de forraje y componentes del rendimiento del pasto Brachiaria humidicola CIAT 6133 con diferente manejo de la defoliación. Téc Pecu Méx 46(4):427-438.

Mena-Urbina, M.A. Hernández-Garay, A., Enríquez-Quiroz, J.F., PérezPérez, J., Zaragoza-Ramírez, J.L., Velasco-Zebadua M.E., y Avellaneda-Cevallos J. 2007. Efecto de asignaciones de forraje, en pastoreo, sobre pasto Insurgente y producción de vaquillas en el trópico húmedo. Agrociencia 41: 1-12.

Murillo, F.J.C. 1999. Respuesta de una pradera de estrella (Cynodon nlemfuencis), bermuda (Cynodon dactylon) y guinea (Panicum máximum), a un sistema de pastoreo intensivo tecnificado móvil con bovinos de engorda. Tesis de Maestría, Universidad de Colima, Colima, México.

Olivera, Y., Machado, R., y del Pozo, P.P. 2006. Características botánicas y agronómicas de especies forrajeras importantes del género Brachiaria. Pastos y Forrajes 29(1):1-5.

Olivera, Y., y Manchado R. 2004. Evaluación de especies del género Brachiaria en suelos ácidos e infértiles durante la época de mínimas precipitaciones. Pastos y Forrajes. 27(3):225-226. 
Comportamiento Forrajero de Brachiaria decumbens pastoreada con ovinos Pelibuey a diferentes niveles de asignación en el trópico húmedo del Ecuador

Paladines, M.O. 1992. Metodología de pastizales para trabajar en fincas y proyectos de desarrollo agropecuario. Proyecto de Fomento Ganadero - PROFOGAN. Convenio Ecuatoriano Alemán. Serie Metodológica, Manual No 1. Pasturas y Forrajes. FEPP. Quito.

Passoni, F., Rosemberg, M., y Flores, A. 1992. Evaluación de gramíneas y leguminosas forrajeras en Satipo, Perú. Pasturas Tropicales 14(1):32-35.

Pueyo, J., Lacopiño, L., Fonseca, J., Burns, J., y Mansilla, A. 2008. Medición de la producción primaria del pastizal natural en el centro norte de la provincia de Entre Rios. Red de ensayos. Estación Experimental Agropecuaria Paraná, INTA. p 1-9. Consultado el 20 de julio del 2013. http://www.elsitioagricola. com/gacetillas/concepci on/co2005gacetillas/pastizales.asp.

Ramírez, R.O., Pérez P.J., Hernández G.A.A., Herrera H.J.G., y Martínez H.P.A. 2003. Evaluación del rendimiento y la utilización de la asociación estrella-clitoria cosechada a diferente asignación de forraje. Tec Pecu Mex 41(2):219-230.

Rincón-Carruyo, X.R., Clavero, T.J., Rincón, U.E., Quintero, C.F., y Márquez, A.T. 1997. Evaluación de parámetros agronómicos y fisiológicos en cuatro cultivares de pasto Buffel (Cenchrus ciliaris L.) en macetas. II. Planta adulta. Rev. Fac. Agron. (LUZ). $14: 649-656$.

Rodrigues, R.C., de Oliveira Souza Lima, D., da Silva Cabral, L., de Melo Plese, L.P., Lacerda Matos Pereira Scaramuzza, W., Alves Utsonomya, T.C., Costa de Siqueira, J., y Ribeiro de Jesús, A.P. 2012. Produção e morfofisiologia do capim Brachiaria brizantha cv. Xaraés sob doses de nitrogênio e fósforo. Revista Brasileira de Agropecuária Sustentável. 2(1):124-131.

Rojas-Hernández, S., Olivares-Pérez, J., Jiménez-Guillén, R., GutiérrezSegura, I., y Aviléz-Nova, F. 2011. Producción de materia seca y componentes morfológicos de cuatro cultivares de Brachiaria en el trópico. Avances en Investigación Agropecuaria 15(1):3-8.

Vázquez, B., Fernández, K., y Rodríguez, Y. 2010. Caracterización del comportamiento Micorrízico en Brachiaria decumbens inoculada con Glamus Hui-like. Cultivos Tropicales. 31(3):58-59.
Figura 1. Diseño de campo de las parcelas experimentales.

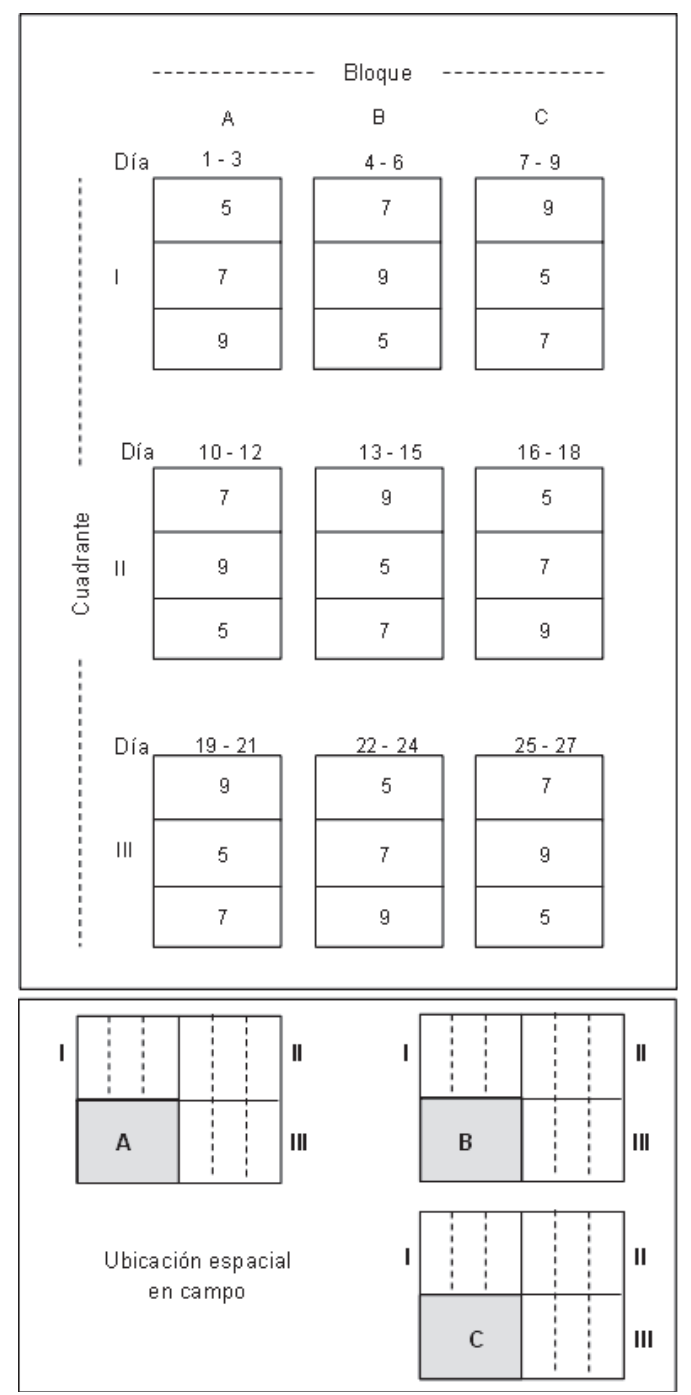

Cuadro 1. Producción de materia seca de forraje en los periodos de rebrote (PR) de la época seca por efecto de las asignaciones (t ha-1).

\begin{tabular}{|c|c|c|c|c|c|c|c|c|}
\hline \multirow[b]{2}{*}{ PR } & \multicolumn{3}{|c|}{---- Asignación forrajera, \% } & \multirow{2}{*}{$\bar{X}_{P R}$} & \multicolumn{3}{|c|}{--- Asignación forrajera, \% } & \multirow{2}{*}{$\overline{\bar{X}}_{P R}$} \\
\hline & 5 & 7 & 9 & & 5 & 7 & 9 & \\
\hline & \multicolumn{4}{|c|}{ Parte aérea } & \multicolumn{4}{|c|}{ Tallos } \\
\hline 1 & $0,77^{\mathrm{a}}$ & $0,74^{\mathrm{a}}$ & $0,96^{\mathrm{a}}$ & $0,82^{a}$ & $0,17^{\mathrm{a}}$ & $0,17^{\mathrm{a}}$ & 0,18 & $0,17^{\mathrm{a}}$ \\
\hline 2 & $0,23^{\mathrm{a}}$ & $0,27^{\mathrm{a}}$ & $0,38^{b}$ & $0,29^{b}$ & $0,04^{\mathrm{a}}$ & $0,05^{\mathrm{a}}$ & $0,08^{\mathrm{a}}$ & $0,06^{b}$ \\
\hline \multirow[t]{2}{*}{$\Delta(\%)$} & 70,10 & 63,50 & 60,40 & 64,40 & 76,50 & 70,60 & 55,60 & 67,30 \\
\hline & \multicolumn{4}{|c|}{ Láminas foliares } & \multicolumn{4}{|c|}{ Vainas } \\
\hline 1 & $0,40^{\mathrm{a}}$ & $0,37^{\mathrm{a}}$ & $0,53^{\mathrm{a}}$ & $0,43^{\mathrm{a}}$ & $0,20^{\mathrm{a}}$ & $0,19^{\mathrm{a}}$ & $0,26^{\mathrm{a}}$ & $0,22^{\mathrm{a}}$ \\
\hline 2 & $0,13^{a}$ & $0,15^{\mathrm{a}}$ & $0,20^{b}$ & $0,16^{\mathrm{b}}$ & $0,06^{a}$ & $0,07^{\mathrm{a}}$ & $0,10^{b}$ & $0,08^{b}$ \\
\hline$\Delta(\%)$ & 67,50 & 59,50 & 62,30 & 63,10 & 70,00 & 63,20 & 61,50 & 64,60 \\
\hline
\end{tabular}

Valores dentro de hilera con letras distintas indican diferencias $a=0,05 ; \Delta$ Porcentaje que disminuyó en el segundo PR. 
Figura 2. Efectos simples y producción acumulada de las asignaciones en la producción de la parte aérea, tallos, láminas foliares y vainas y producción acumulada en ambos periodos de rebrote.

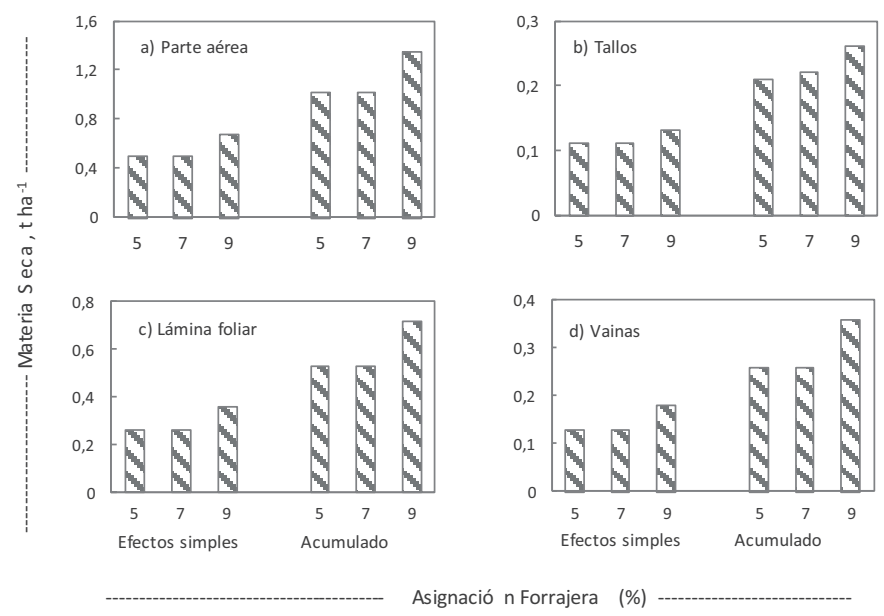

Cuadro 2. Efecto de las asignaciones forrajeras en el porcentaje de vainas en los periodos de rebrote.

\begin{tabular}{crrrr}
\hline & \multicolumn{3}{c}{ Asignaciones forrajeras (\%) } & $\bar{X}$ \\
\cline { 2 - 4 } PR & 5 & 7 & 9 & PR \\
\hline 1 & $25,07^{a}$ & $25,29^{a}$ & $26,69^{b}$ & 25,68 \\
2 & $26,31^{a}$ & $28,09^{a}$ & $30,13^{b}$ & 28,18 \\
$\bar{X}$ Asignaciones & $25,69^{a}$ & $26,69^{a}$ & $28,41^{b}$ & \\
$\Delta(\%)$ & $1,20^{a}$ & $2,80^{a}$ & $3,40^{b}$ & \\
\hline
\end{tabular}

Valores con letras distintas dentro de hilera indican diferencias con DMS $a=0,05$ para el $9 \%$; letras mayúsculas se leen para el segundo PR con Tukey $a=0,05$;

Figura 3. Efecto de las asignaciones en el porcentaje de materia seca de tallos y láminas foliares en el total de la parte aérea durante los periodos de rebrote.

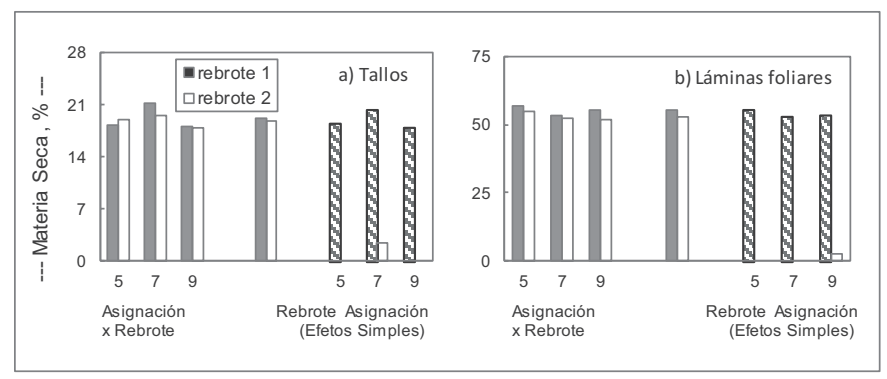

